\title{
Dissolution Behaviours of Acetaminophen and Ibuprofen Tablet Influenced By L- HPC 21, 22, and Sodium Starch Glycolate as Disintegrant
}

\author{
Yoga W. Wardhana*, Dradjad Priambodo \\ Department Pharmaceutics and Pharmaceuticals Technology Faculty of Pharmacy, Universitas \\ Padjadjaran \\ Jl. Raya Bandung - Sumedang Km. 21 Jatinangor 45363, Sumedang, Indonesia
}

Received: 5 Sep 2019/Revised: 23 Sep 2019/Accepted: 23 Sep 2019/Published: 1 Oct 2019

\begin{abstract}
The dissolution of tablets is one of a drug absorption determinant. Disintegrant agent has play an important role on determining the dissolution of tablets. In this experiment, the dissolution behaviours of Acetaminophen and Ibuprofen Tablet was studied using various disintegrant agent such as Low substituted - Hydroxypropyl Cellulose (L-HPC) 21, L-HPC 22 and Sodium Starch Glycolate (SSG) as comparator. Those disintegrant agents were used at three concentration $(6 \%, 7 \%$ and $8 \%)$ for every tablets formula. Tablets were made by wet granulation method and pressed using single punch 13 $\mathrm{mm}$ flat E. Korsch machine. Evaluation of each tablets quality were conducted include for uniformity of weight and size (diameter and thickness), hardness, friability, disintegration time and dissolution. Physically standards from tablets were in good condition, the standards of the weight and thickness uniformity, hardness and friability met the requirement. The dissolution profile on Acetaminophen Tablets showed that only tablet with $6 \% \mathrm{~L}-\mathrm{HPC} 21$ did not meet the requirement of FI V (Q $=80 \%$, 30 minutes), but on Ibuprofen Tablets where met the requirement of FI V ( $\mathrm{Q}=80 \%, 60$ minutes) only tablet with $8 \%$ L- HPC $21,7 \%$ and $8 \%$ SSG. The conclusion of the study was the L-HPC has more disintegrant character at hydrophilic active ingredients.
\end{abstract}

Keywords: Acetaminophen Tablet, Ibuprofen Tablet, SSG, L-HPC 21 and 22, Dissolution Profile

\section{Introduction}

Tablet dosage forms has widely used in global market cause of all advantages and benefits. The composition of all compressed tablets should, in fact, be designed to guarantee that they will readily undergo both disintegration and dissolution in the upper gastrointestinal (GI) tract.

Dissolution tests are used nowadays in a wide variety of applications: to help identify which formulations will produce the best results in the clinic, to release products to the market, to verify batch to batch reproducibility, and to help identify whether changes made to formulations or their manufacturing procedure after marketing approval are likely to affect the performance in the clinic. Further, dissolution tests can sometimes be implemented to help determine whether a generic version of the medicine can be approved or rejected [3].
Dissolution tests can be used to predict the in vivo performance of the dosage form when release of the drug is the limiting factor in the absorption process. The drug release profile influenced by complex factors, one of all is excipient selection in tablet formulation [3].

Standard in vitro dissolution testing models include two processes: the release of drug substance from the solid dosage form and drug dissolution. Drug release is determined by formulation factors such as disintegration/dissolution of formulation excipients or drug diffusion through the formulation. Drug dissolution is affected by the physicochemical substance properties (e.g., solubility, diffusivity), solid-state pro-perties of the substance (e.g., particle surface area, polymorphism), and formula-tion properties (e.g., wetting, solubilization). In vitro dissolution testing should thus provide predictions of both the drug release and the dissolution processes in vivo. To reach this goal, the choice of 
dissolution apparatus be carefully considered and test medium should [6].

Disintegration/dissolution by formulation factors effect from excipient which adding as disintegrant such as Low substituted hydroxypropyl cellulose (L- HPC) and Sodium starch glycolate (SSG). $\mathrm{L}-\mathrm{HPC}$ is widely used in oral solid dosage forms. It is primarily used as a disintegrant, and as a binder for tablets and granules in wet or dry granulation. Whereas SSG commonly is used in oral pharmaceuticals as a disintegrant in capsule and tablet formulations. Usually disintegration occurs by rapid uptake of water followed by rapid and enormous swelling [7].

In this experiment, the investigation of $\mathrm{L}-$ HPC 21, 22 and SSG effect as disintegrant has been compared between hydrophilic substance such as Acetaminophen Tablet and hydrophobic substance such as Ibuprofen Tablet in the differentiation dissolution profiles. The result of this experiment can be used by formulator pharmacist as recommendation in designing solid dosage formulation.

\section{Materials and Methods}

\subsection{Materials}

Acetaminophen Ex Hebei (from Pharmacy Distributor PT. Bratachem), Ibuprofen (kindly provided by PT. Indofarma Tbk.), Low-Substituted Hydroxy propyl Cellulose (L-HPC) 21 and $22 \mathrm{Ex}$ Shin-Etsu Chemical Co., Ltd. (kindly provided by PT. Lawsim Zecha), Sodium Starch Glycolate as Primojel Ex Gujarat Microwax Ltd., Starch for tablet / Ampro-tab (generously donated from PT. Holi Farma), Potassium dihydrogen Phosphat, Silicon dioxyde as Aerosil and Magnesium Stearate (from Pharmacy Distributor CV. Quadrant Lab.), Talcum (generously donated from PT. Kimia Farma), NaOH (from Pharmacy Distributor PT. Bratachem), Aquadest.

\subsection{Methods}

\section{Tablet Formulation}

The tablets was made in variation formulas shown in Table 1 and 2. The preparations were using wet granulation with 10 mesh for wet mass granulator filter and 16 mesh for drying mass

Table 1. Composition of tablet excipients in Acetaminophen Tablets

\begin{tabular}{cccccccccc}
\hline \multirow{2}{*}{ Ingredients } & \multicolumn{3}{c}{$\mathrm{F}_{\mathrm{A}}(\%)$} & \multicolumn{3}{c}{$\mathrm{F}_{\mathrm{B}}(\%)$} & \multicolumn{3}{c}{$\mathrm{F}_{\mathrm{C}}(\%)$} \\
& $\mathrm{FA}_{1}$ & $\mathrm{FA}_{2}$ & $\mathrm{FA}_{3}$ & $\mathrm{FB}_{1}$ & $\mathrm{FB}_{2}$ & $\mathrm{FB}_{3}$ & $\mathrm{FC}_{1}$ & $\mathrm{FC}_{2}$ & $\mathrm{FC}_{3}$ \\
\hline Acetaminophen & 77 & 77 & 77 & 77 & 77 & 77 & 77 & 77 & 77 \\
Lactose & 11.2 & 10.2 & 9.2 & 11.2 & 10.2 & 9.2 & 11.2 & 10.2 & 9.2 \\
Amylum for Paste & 3.3 & 3.3 & 3.3 & 3.3 & 3.3 & 3.3 & 3.3 & 3.3 & 3.3 \\
L-HPC 21 & 6 & 7 & 8 & - & - & - & - & - & - \\
L-HPC 22 & - & - & - & 6 & 7 & 8 & - & - & - \\
Primojel & - & - & - & - & - & - & 6 & 7 & 8 \\
Mg Stearate & 1 & 1 & 1 & 1 & 1 & 1 & 1 & 1 & 1 \\
Aerosil & 0.5 & 0.5 & 0.5 & 0.5 & 0.5 & 0.5 & 0.5 & 0.5 & 0.5 \\
Talcum & 1 & 1 & 1 & 1 & 1 & 1 & 1 & 1 & 1 \\
\hline
\end{tabular}

Table 2. Composition of tablet excipients in Ibuprofen Tablets

\begin{tabular}{cccccccccc}
\hline Ingredients & \multicolumn{3}{c}{$\mathrm{P}_{\mathrm{A}}(\%)$} & \multicolumn{3}{c}{$\mathrm{P}_{\mathrm{B}}(\%)$} & \multicolumn{3}{c}{$\mathrm{P}_{\mathrm{C}}(\%)$} \\
& $\mathrm{P}_{\mathrm{A} 1}$ & $\mathrm{P}_{\mathrm{A} 2}$ & $\mathrm{P}_{\mathrm{A} 3}$ & $\mathrm{P}_{\mathrm{B} 1}$ & $\mathrm{P}_{\mathrm{B} 2}$ & $\mathrm{P}_{\mathrm{B} 3}$ & $\mathrm{P}_{\mathrm{C} 1}$ & $\mathrm{P}_{\mathrm{C} 2}$ & $\mathrm{P}_{\mathrm{C} 3}$ \\
\hline Ibuprofen & 61.5 & 61.5 & 61.5 & 61.5 & 61.5 & 61.5 & 61.5 & 61.5 & 61.5 \\
Lactose & 18.75 & 17.75 & 16.75 & 18.75 & 17.75 & 16.75 & 18.75 & 17.75 & 16.75 \\
Amylum for Paste & 11.25 & 11.25 & 11.25 & 11.25 & 11.25 & 11.25 & 11.25 & 11.25 & 11.25 \\
L-HPC 21 & 6 & 7 & 8 & - & - & - & - & - & - \\
L-HPC 22 & - & - & - & 6 & 7 & 8 & - & - & - \\
Primojel & - & - & - & - & - & - & 6 & 7 & 8 \\
Mg Stearate & 1 & 1 & 1 & 1 & 1 & 1 & 1 & 1 & 1 \\
Aerosil & 0.5 & 0.5 & 0.5 & 0.5 & 0.5 & 0.5 & 0.5 & 0.5 & 0.5 \\
Talcum & 1 & 1 & 1 & 1 & 1 & 1 & 1 & 1 & 1 \\
\hline
\end{tabular}


granulator sieving. Then, after manually mixture with disintegrants and lubricants for just 3 minutes, all of granules were evaluation to determine that all of granule ready to compress.

\section{Granules Evaluation}

Evaluation for granules quality were include loss on drying, bulk density, tap density, compressibility, flow ability and angle of requirements that has a good criteria such as good compressibility and smooth fluently. Then all can be pressed into tablet.

\section{Tablet Evaluation}

After all of granules passed from evaluation, granules will be pressed by $13 \mathrm{~mm}$ flat single punch E. Korsch Tablet Machine. Then quality of tablet will be checked for hardness, friability, thickness, weight and uniformity include disintegration time and dissolution.

\section{Result and Discussion}

The evaluation result of granules quality were found those all of granules met the requirements standard for compressed tablet. Then, all of granules will proceed to pressed into tablet with 13 $\mathrm{mm}$ flat single punch E. Korsch Tablet Machine. After that, tablets was evaluated for checking tablet quality, such as below.

Performance of physic from tablets were in good condition, the standards of the weight and thickness uniformity, hardness and friability met the requirement. But, if looked carefully at disintegration time and solubility percentage (dissolution). There were found something make some formula out of standards.

The results of disintegration time and dissolution shown that only Acetaminophen tablets in formula $\mathrm{F}_{\mathrm{A} 1}$ did not meet the requirement for desintegration time (15 minutes) and dissolution (30 minutes, $80 \%$ ). Found differently at Ibuprofen tablet, all of

Table 3. Granule Evaluation for Compression mass (granules) Acetaminophen Tablets ( $\mathrm{n}=3$ )

\begin{tabular}{|c|c|c|c|c|c|c|c|c|c|}
\hline \multirow{2}{*}{ Parameters } & \multicolumn{3}{|c|}{ L-HPC 21} & \multicolumn{3}{|c|}{ L-HPC 22} & \multicolumn{3}{|c|}{ SSG } \\
\hline & $\mathrm{FA}_{1}$ & $\mathrm{FA}_{2}$ & $\mathrm{FA}_{3}$ & $\mathrm{FB}_{1}$ & $\mathrm{FB}_{2}$ & $\mathrm{FB}_{3}$ & $\mathrm{FC}_{1}$ & $\mathrm{FC}_{2}$ & $\mathrm{FC}_{3}$ \\
\hline $\begin{array}{c}\text { Loss on drying } \\
(\%)\end{array}$ & 1.8 & 0.62 & 0.4 & 1.3 & 1.1 & 0.6 & 0.7 & 0.62 & 0.6 \\
\hline $\begin{array}{l}\text { Bulk density } \\
\qquad(\mathrm{g} / \mathrm{ml})\end{array}$ & $\begin{array}{c}0.551 \pm \\
0.008\end{array}$ & $\begin{array}{c}0.541 \pm \\
0.015\end{array}$ & $\begin{array}{c}0.561 \pm \\
0.008\end{array}$ & $\begin{array}{c}0.532 \pm \\
0.022\end{array}$ & $\begin{array}{c}0.546 \pm \\
0.008\end{array}$ & $\begin{array}{c}0.536 \pm \\
0.008\end{array}$ & $\begin{array}{c}0.582 \pm \\
0.009\end{array}$ & $\begin{array}{c}0.561 \pm \\
0.008\end{array}$ & $\begin{array}{c}0.582 \pm \\
0.009\end{array}$ \\
\hline $\begin{array}{l}\text { Tap density } \\
\qquad(\mathrm{g} / \mathrm{ml})\end{array}$ & $\begin{array}{c}0.606 \pm \\
0.018\end{array}$ & $\begin{array}{c}0.612 \pm \\
0.011\end{array}$ & $\begin{array}{c}0.646 \pm \\
0.021\end{array}$ & $\begin{array}{c}0.594 \pm \\
0.010\end{array}$ & $\begin{array}{c}0.619 \pm \\
0.011\end{array}$ & 0.625 & $\begin{array}{c}0.674 \pm \\
0.013\end{array}$ & 0.625 & $\begin{array}{c}0.674 \pm \\
0.013\end{array}$ \\
\hline $\begin{array}{c}\text { Compressibility } \\
(\%)\end{array}$ & $\begin{array}{c}9.044 \pm \\
4.057\end{array}$ & $\begin{array}{c}11.631 \pm \\
2.929\end{array}$ & $\begin{array}{c}13.027 \pm \\
4.056\end{array}$ & $\begin{array}{l}10.517 \\
\pm 2.252\end{array}$ & $\begin{array}{l}11.735 \\
\pm 1.484\end{array}$ & $\begin{array}{c}14.24 \pm \\
1.386\end{array}$ & $\begin{array}{l}13.632 \\
\pm 1.554\end{array}$ & $\begin{array}{c}10.24 \pm \\
1.386\end{array}$ & $\begin{array}{l}13.632 \pm \\
1.554\end{array}$ \\
\hline $\begin{array}{l}\text { Flow ability } \\
\text { (g/detik) }\end{array}$ & $\begin{array}{c}12.739 \pm \\
1.24\end{array}$ & $\begin{array}{c}10.706 \pm \\
0.68\end{array}$ & $\begin{array}{c}9.206 \pm \\
0.21\end{array}$ & $\begin{array}{l}12.197 \\
\pm 0.958\end{array}$ & $\begin{array}{l}12.056 \\
\pm 1.01\end{array}$ & $\begin{array}{c}10.41 \pm \\
0.45\end{array}$ & $\begin{array}{c}7.267 \pm \\
1.42\end{array}$ & $\begin{array}{c}10.281 \\
\pm 1.9\end{array}$ & $\begin{array}{l}8.61 \pm \\
0.58\end{array}$ \\
\hline $\begin{array}{c}\text { Angle of repose } \\
\left({ }^{\circ}\right)\end{array}$ & $\begin{array}{c}26.087 \pm \\
3.11 \\
\end{array}$ & $\begin{array}{c}23.152 \pm \\
1.02\end{array}$ & $\begin{array}{c}19.587 \pm \\
1.299 \\
\end{array}$ & $\begin{array}{c}23.502 \\
\pm 2.45 \\
\end{array}$ & $\begin{array}{c}20.25 \pm \\
3.37 \\
\end{array}$ & $\begin{array}{c}20.76 \pm \\
1.56 \\
\end{array}$ & $\begin{array}{c}15.69 \pm \\
1.08 \\
\end{array}$ & $\begin{array}{c}19.9 \pm \\
1.85 \\
\end{array}$ & $\begin{array}{c}20.12 \pm \\
2.54\end{array}$ \\
\hline
\end{tabular}

Table 4. Granule Evaluation for Compression mass (granules) Ibuprofen Tablets $(n=3)$

\begin{tabular}{|c|c|c|c|c|c|c|c|c|c|}
\hline \multirow{2}{*}{ Parameters } & \multicolumn{3}{|c|}{ L-HPC 21} & \multicolumn{3}{|c|}{ L-HPC 22} & \multicolumn{3}{|c|}{ SSG } \\
\hline & $\mathrm{PA}_{1}$ & $\mathrm{PA}_{2}$ & $\mathrm{PA}_{3}$ & $\mathrm{~PB}_{1}$ & $\mathrm{~PB}_{2}$ & $\mathrm{~PB}_{3}$ & $\mathrm{PC}_{1}$ & $\mathrm{PC}_{2}$ & $\mathrm{PC}_{3}$ \\
\hline $\begin{array}{c}\text { Loss on drying } \\
(\%)\end{array}$ & 1.64 & 1.61 & 1.94 & 1.47 & 1.48 & 1.55 & 1.76 & 1.71 & 1.77 \\
\hline $\begin{array}{l}\text { Bulk density } \\
\qquad(\mathrm{g} / \mathrm{ml})\end{array}$ & $\begin{array}{c}0.449 \pm \\
0.005\end{array}$ & $\begin{array}{c}0.442 \pm \\
0.003\end{array}$ & $\begin{array}{c}0.444 \pm \\
0.005\end{array}$ & $\begin{array}{c}0.478 \pm \\
0.059\end{array}$ & $\begin{array}{c}0.427 \pm \\
0.008\end{array}$ & $\begin{array}{c}0.454 \pm \\
0.005\end{array}$ & $\begin{array}{c}0.452 \pm \\
0.003\end{array}$ & $\begin{array}{c}0.457 \pm \\
0.003\end{array}$ & $\begin{array}{r}0.472 \pm \\
0.003\end{array}$ \\
\hline $\begin{array}{l}\text { Tap density (g/ } \\
\text { ml) }\end{array}$ & $\begin{array}{c}0.557 \pm \\
0.004\end{array}$ & $\begin{array}{c}0.557 \pm \\
0.012\end{array}$ & $\begin{array}{c}0.560 \pm \\
0.012\end{array}$ & $\begin{array}{c}0.544 \pm \\
0.004\end{array}$ & $\begin{array}{c}0.533 \pm \\
0.007\end{array}$ & $\begin{array}{c}0.555 \pm \\
0.008\end{array}$ & $\begin{array}{c}0.555 \pm \\
0.008\end{array}$ & $\begin{array}{c}0.557 \pm \\
0.004\end{array}$ & $\begin{array}{r}0.576 \pm \\
0.004\end{array}$ \\
\hline $\begin{array}{l}\text { Compressibility } \\
(\%)\end{array}$ & $\begin{array}{c}19.44 \pm \\
1.172\end{array}$ & $\begin{array}{c}20.71 \pm \\
1.762\end{array}$ & $\begin{array}{c}20.77 \pm \\
0.905\end{array}$ & $\begin{array}{c}18.86 \pm \\
0.968\end{array}$ & $\begin{array}{c}19.47 \pm \\
0.739\end{array}$ & $\begin{array}{c}18.18 \pm \\
0.210\end{array}$ & $\begin{array}{c}18.47 \pm \\
0.725\end{array}$ & $\begin{array}{c}17.88 \pm \\
0.637\end{array}$ & $\begin{array}{r}18.11 \pm \\
0.627\end{array}$ \\
\hline $\begin{array}{l}\text { Flow ability } \\
\text { (g/detik) }\end{array}$ & $\begin{array}{c}12.32 \pm \\
1.698\end{array}$ & $\begin{array}{c}12.92 \pm \\
0.685\end{array}$ & $\begin{array}{c}11.31 \pm \\
0.593\end{array}$ & $\begin{array}{c}12.96 \pm \\
0.897\end{array}$ & $\begin{array}{c}12.68 \pm \\
0.281\end{array}$ & $\begin{array}{c}10.91 \pm \\
0.422\end{array}$ & $\begin{array}{c}12.86 \pm \\
0.102\end{array}$ & $\begin{array}{c}12.76 \pm \\
0.764\end{array}$ & $\begin{array}{r}13.08 \pm \\
0.987\end{array}$ \\
\hline Angle of repose & $\begin{array}{c}24.49 \pm \\
0.293\end{array}$ & $\begin{array}{c}24.32 \pm \\
0.145\end{array}$ & $\begin{array}{c}24.64 \pm \\
0.808\end{array}$ & $\begin{array}{c}24.15 \pm \\
1.004\end{array}$ & $\begin{array}{c}25.87 \pm \\
0.612\end{array}$ & $\begin{array}{c}25.41 \pm \\
0.166\end{array}$ & $\begin{array}{c}24.71 \pm \\
0.956\end{array}$ & $\begin{array}{c}23.61 \pm \\
0.910\end{array}$ & $\begin{array}{r}26.00 \pm \\
0.121\end{array}$ \\
\hline
\end{tabular}


tablet were not passed from desintegration time but on the dissolution only formula $\mathrm{P}_{\mathrm{A} 3}, \mathrm{P}_{\mathrm{C} 2}$ and $\mathrm{P}_{\mathrm{C} 3}$ were passed from the test (60 minutes, $80 \%$ ). This results were concluded that L-HPC 21 has more desintegrant character than L-HPC 22 especially with hydrophobic API.

\section{Conclusion}

From the result shown that Aceta-minophen tablets with $6 \% \mathrm{~L}-\mathrm{HPC} 21$ did not meet the requirement of FI V (Q $=80 \%, 30$ minutes), but on Ibuprofen Tablets where met the requirement of FI V $(\mathrm{Q}=$ $80 \%, 60$ minutes) only for tablet with $8 \%$ L-HPC

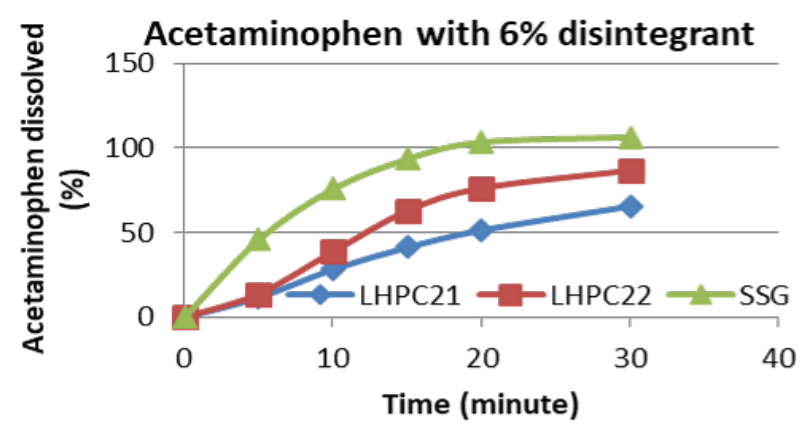

(a)

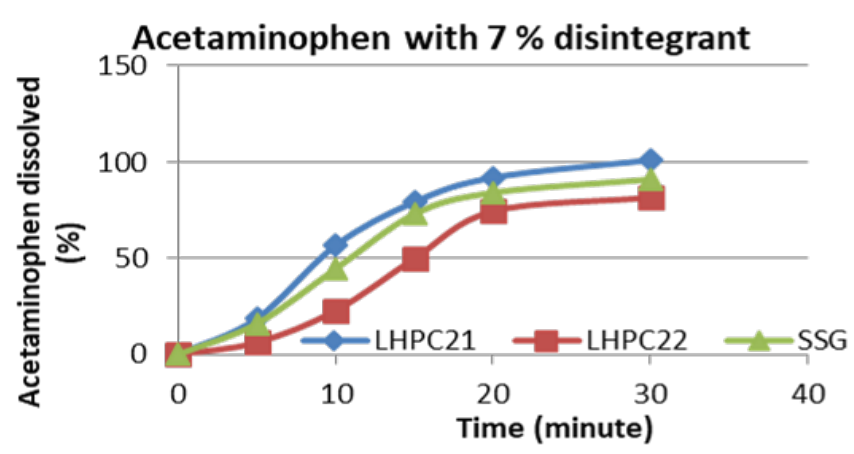

(c)

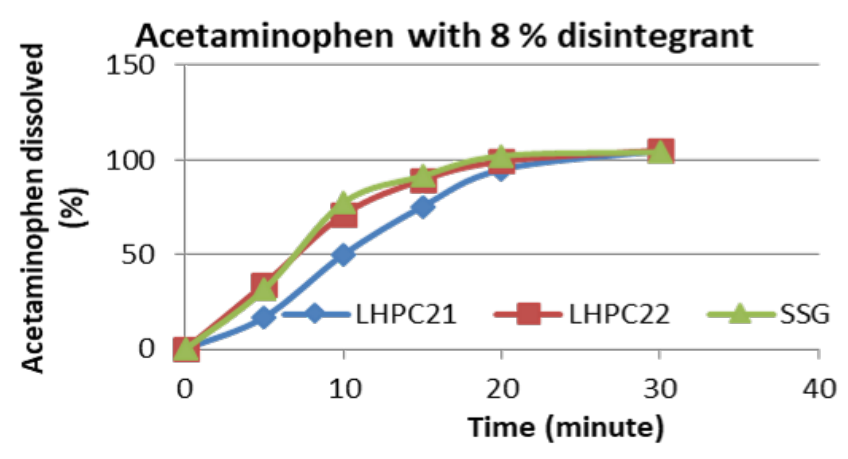

(e)
21, $7 \%$ and $8 \%$ SSG. Based on those data, its were concluded that L-HPC has more disintegrant character with hydrophilic active ingredients. Between those L-HPC, L-HPC 21 has more desintegrant nature than L-HPC 22.

\section{Acknowledgement}

The authors would like to thanks to PT. Lawsim Zecha, PT. Holi Farma, PT. Kimia Farma and PT. Indofarma for their kindly materials provided, and to Irene Natalie and Randy Andrian Herawan for the data collection.

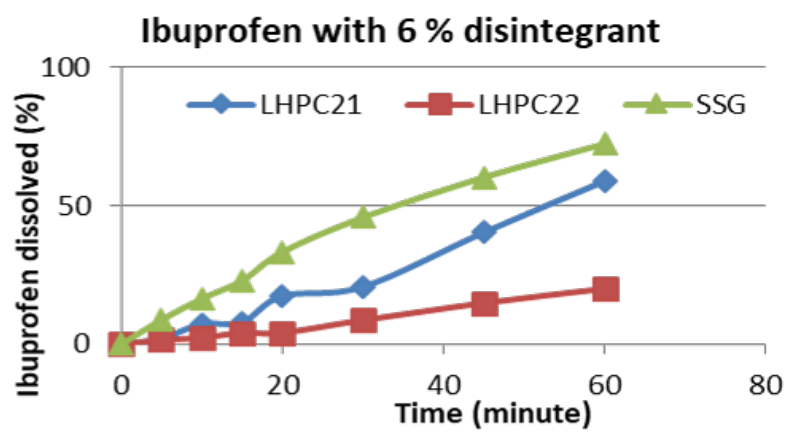

(b)

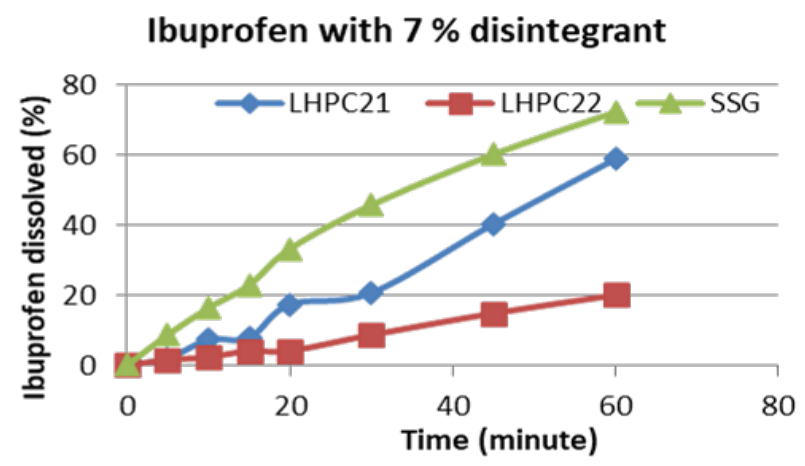

(d)

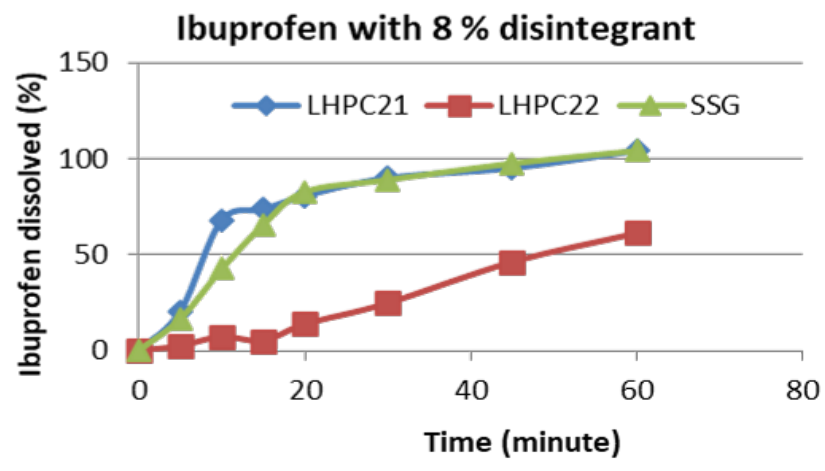

(f)

Figure 1. Dissolution Profile of Acetaminophen and Ibuprofen Tablets in various disintegrant percentation $(\mathrm{n}=3)$ 
Table 5. Evaluation for Acetaminophen Tablets $(\mathrm{n}=20)$

\begin{tabular}{lccccccccc}
\hline \multirow{2}{*}{ Parameters } & \multicolumn{3}{c}{ L-HPC 21} & \multicolumn{3}{c}{ L-HPC 22 } & \multicolumn{3}{c}{ SSG } \\
& $\mathrm{FA}_{1}$ & $\mathrm{FA}_{2}$ & \multicolumn{1}{c}{$\mathrm{FA}_{3}$} & $\mathrm{FB}_{1}$ & $\mathrm{FB}_{2}$ & $\mathrm{FB}_{3}$ & $\mathrm{FC}_{1}$ & $\mathrm{FC}_{2}$ & $\mathrm{FC}_{3}$ \\
\hline Weight Uniformity & $641.15 \pm$ & $662.68 \pm$ & $630.52 \pm$ & 650.87 & 641.58 & $638.2 \pm$ & $652.4 \pm$ & $648.2 \pm$ & $655.09 \pm$ \\
(mg) & 19.77 & 8.15 & 12.22 & \pm 24.72 & \pm 10.2 & 8.48 & 10.84 & 10.81 & 15.45 \\
Thickness Uniformity & $5.352 \pm$ & $5.501 \pm$ & $5.308 \pm$ & $5.458 \pm$ & $5.422 \pm$ & $5.425 \pm$ & $5.349 \pm$ & $5.244 \pm$ & $5.354 \pm$ \\
(mm) & 0.236 & 0.059 & 0.089 & 0.155 & 0.053 & 0.076 & 0.083 & 0.085 & 0.072 \\
& $106 \pm$ & $83.25 \pm$ & $92 \pm$ & $103.4 \pm$ & $86.6 \pm$ & $93 \pm$ & $109 \pm$ & 55.775 & $133.375 \pm$ \\
Hardness (N) & 30.75 & 9.92 & 17.85 & 19.63 & 8.34 & 19.13 & 26.37 & \pm 5.43 & 18.96 \\
& $0.448 \pm$ & $0.456 \pm$ & $0.342 \pm$ & $0.394 \pm$ & $0.604 \pm$ & $0.472 \pm$ & $0.691 \pm$ & $0.78 \pm$ & $0,679 \pm$ \\
Friability (\%) & 0.076 & 0.012 & 0.087 & 0.035 & 0.198 & 0.071 & 0.151 & 0.345 & 0.038 \\
Desintegration time & 18.061 & 12.361 & 11.061 & 12.261 & 11.955 & 2.05 & 8.322 & 8.411 & 4.533 \\
(minutes) & & & & & & & & & \\
Dissolution (\%) & 65.642 & 101.065 & 104.924 & 86.822 & 81.577 & 105.21 & 106.39 & 91.064 & 104.098 \\
\hline
\end{tabular}

Table 6. Evaluation for Ibuprofen Tablets $(\mathrm{n}=20)$

\begin{tabular}{lccccccccc}
\hline \multirow{2}{*}{ Parameters } & \multicolumn{3}{c}{ L-HPC 21 } & \multicolumn{3}{c}{ L-HPC 22 } & \multicolumn{3}{c}{$\mathrm{SSG}$} \\
& $\mathrm{PA}_{1}$ & $\mathrm{PA}_{2}$ & $\mathrm{PA}_{3}$ & $\mathrm{~PB}_{1}$ & $\mathrm{~PB}_{2}$ & $\mathrm{~PB}_{3}$ & $\mathrm{PC}_{1}$ & $\mathrm{PC}_{2}$ & $\mathrm{PC}_{3}$ \\
\hline Weight & $660.8 \pm$ & $657.8 \pm$ & $648.2 \pm$ & $655.3 \pm$ & $649.2 \pm$ & $658.5 \pm$ & $654 \pm$ & $646.6 \pm$ & $649.4 \pm$ \\
Uniformity (mg) & 4.188 & 5.053 & 6.709 & 6.052 & 3.876 & 9.327 & 3.005 & 2.377 & 6,459 \\
Thickness & $4.217 \pm$ & $4.23 \pm$ & $4.23 \pm$ & $4.23 \pm$ & $4.23 \pm$ & $4.22 \pm$ & $4.24 \pm$ & $4.22 \pm$ & $4.24 \pm$ \\
Uniformity (mm) & 0.050 & 0.06 & 0.06 & 0.04 & 0.05 & 0.05 & 0.04 & 0.03 & 0.06 \\
Hardness (N) & $120 \pm$ & $124 \pm$ & $105.3 \pm$ & $116.9 \pm$ & $125.9 \pm$ & $113.1 \pm$ & $115.7 \pm$ & $123,1 \pm$ & $105.3 \pm$ \\
& 14.42 & 15.111 & 11.59 & 14.911 & 11.938 & 15.598 & 16.163 & 15.355 & 11.594 \\
Friability (\%) & $0.47 \pm$ & $0.47 \pm$ & $0.41 \pm$ & $0.47 \pm$ & $0.45 \pm$ & $0.48 \pm$ & $0.67 \pm$ & $0,51 \pm$ & $0,67 \pm$ \\
& 0.03 & 0.26 & 0.03 & 0.02 & 0.05 & 0.04 & 0.08 & 0.01 & 0.05 \\
Desintegration & 59.35 & 48.33 & 38.19 & 51.5 & 42.2 & 33.45 & 25.75 & 11.83 & 10.66 \\
time (minutes) & & & & & & & & & \\
Dissolution (\%) & 58.834 & 79.714 & 104.112 & 20.035 & 43.403 & 61.143 & 72,238 & 96.754 & 104,08 \\
\hline
\end{tabular}

\section{References}

[1] Aulton, M.E., Pharmaceutics: The Science of Dosage Form Design. New York: Longmann Group Churchill Livingstone. p. 234-238, 240242. 2002

[2] Chakraborty, S., Khandai, M., Singh, S. P., and Patra, N. Comparative Study on Effect of Natural and Synthetic Superdisintegrants in the Formulation of Fast Dissolving Tablets. International Journal of Green Pharmacy. 2007; 2(1) : 2225.

[3] Dressman J., Kramer, J., Pharmaceutical Dissolution Testing, Florida: Taylor \& Francis Group, LLC. 2005.

[4] Dressman, J.B., Oral Drug Absorption (Prediction and Assessment), New York:
Marcel Dekker, Inc,. 2000.

[5] Departemen Kesehatan Republik Indonesia. Farmakope Indonesia. Edisi V. Jakarta : Departemen Kesehatan Republik Indonesia. 2014.

[6] Gibson, M., Pharmaceutical Preformulation and Formulation : A Practical Guide from Candidate Drug Selection to Commercial Dosage Form 2nd ed., New York: Informa Healthcare USA, Inc. 2009.

[7] Rowe, Raymond C., Sheskey. Paul J., Quinn, M.E., Handbook of Pharmaceutical Excipients 6th ed., London: RPS Publishing. 2009.

[8] Shin-Etsu Chemical Co. L-HPC : LowSubstituted Hydroxypropyl Cellulose, NF. Japan: Shin-Etsu Chemical Co. p. 4-6. 2008. 